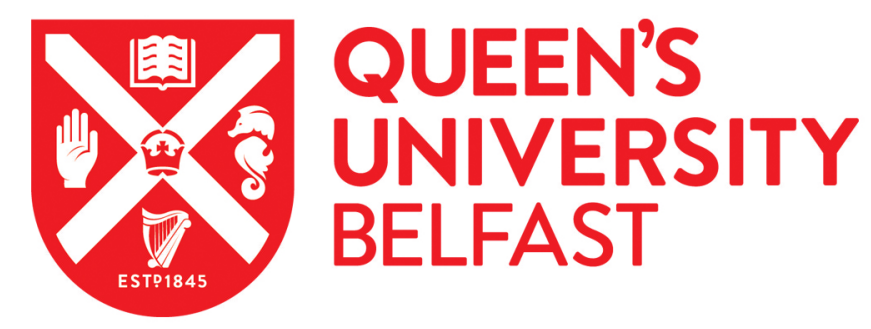

\title{
A Time Series Based Study of Correlation, Channel Power Imbalance and Diversity Gain in Indoor Distributed Antenna Systems at $60 \mathrm{GHz}$
}

Zhang, L., Cotton, S. L., Yoo, S. K., Ngo, H. Q., Fernandez, M., \& Scanlon, W. G. (2021). A Time Series Based Study of Correlation, Channel Power Imbalance and Diversity Gain in Indoor Distributed Antenna Systems at 60 GHz. IEEE Transactions on Antennas and Propagation. https://doi.org/10.1109/TAP.2021.3076171

Published in:

IEEE Transactions on Antennas and Propagation

Document Version:

Peer reviewed version

Queen's University Belfast - Research Portal:

Link to publication record in Queen's University Belfast Research Portal

Publisher rights

Copyright 2021, IEEE.

This work is made available online in accordance with the publisher's policies. Please refer to any applicable terms of use of the publisher.

\section{General rights}

Copyright for the publications made accessible via the Queen's University Belfast Research Portal is retained by the author(s) and / or other copyright owners and it is a condition of accessing these publications that users recognise and abide by the legal requirements associated with these rights.

Take down policy

The Research Portal is Queen's institutional repository that provides access to Queen's research output. Every effort has been made to ensure that content in the Research Portal does not infringe any person's rights, or applicable UK laws. If you discover content in the Research Portal that you believe breaches copyright or violates any law, please contact openaccess@qub.ac.uk. 


\title{
A Time Series Based Study of Correlation, Channel Power Imbalance and Diversity Gain in Indoor Distributed Antenna Systems at $60 \mathrm{GHz}$
}

\author{
Lei Zhang, Simon L. Cotton, Senior Member, IEEE, \\ Seong Ki Yoo, Member, IEEE, Hien Quoc Ngo, Senior Member, IEEE, \\ Marta Fernández, Member, IEEE, and William G. Scanlon, Fellow, IEEE
}

\begin{abstract}
In this paper, we investigate the potential enhancements in signal reliability which can be achieved by using a millimeter-wave distributed antenna system (DAS) within an indoor environment. To achieve this, we measured the signal power simultaneously received at 9 ceiling mounted access point (AP) locations likely to be used in future indoor DAS deployments while a mobile user imitated making a voice call on a hypothetical user equipment. Key metrics, associated with the performance of multiple antenna systems, such as the cross correlation coefficient (CCC) and channel power imbalance (CPI) are determined. It was found that line-of-sight (LOS) and quasi-LOS (QLOS) links with the APs typically led to higher CCC values than the non-LOS (NLOS) cases. Similarly, LOS and QLOS links typically produced higher CPI values between APs than the NLOS case. To enable the reproduction of our results, we have successfully employed autoregressive moving average and autoregressive integrated moving average modeling to the CCC and CPI time series. The performance improvement that can be achieved using a DAS instead of a single AP was evaluated using three commonly deployed diversity combining schemes, namely, selection combining, equal gain combining and maximal ratio combining along with three AP selection mechanisms, namely, per-sample random AP selection, one-shot AP selection and persample optimal AP selection. Finally, we have provided some useful insights into the influence of differing AP numbers on the diversity gain when considering the aforementioned AP selection methods.
\end{abstract}

Index Terms-Autoregressive integrated moving average (ARIMA) modeling, autoregressive moving average (ARMA) modeling, channel measurement, channel power imbalance, distributed antenna system, diversity gain, millimeter wave, time series analysis.

\section{INTRODUCTION}

L. Zhang, S. L. Cotton, and H. Q. Ngo are with the Centre for Wireless Innovation, Institute of Electronics, Communications and Information Technology, Queen's University Belfast, BT3 9DT, UK. E-mail: \{lzhang27, simon.cotton, hien.ngo\}@qub.ac.uk.

S. K. Yoo is with the School of Computing, Electronics, and Mathematics, Coventry University, CV1 5FB, UK. E-mail: ad3869@ coventry.ac.uk.

M. Fernández is with the Communications Engineering Department, University of the Basque Country, 48013 Bilbao, Spain. E-mail: martafernandez010@gmail.com.

W. G. Scanlon is with Tyndall National Institute, Cork T12R5CP, Ireland. E-mail: w.scanlon@ieee.org.

This work was funded in part by the Northern Ireland Department for the Economy as part of the US-Ireland NEMOs project (USI 080). The work of H. Q. Ngo was supported by the U.K. Research and Innovation Future Leaders Fellowships under Grant MR/S017666/1.
$\mathbf{M}$ Illimeter wave (mmWave) technologies are emerging as strong candidates to meet the high data rate demands of fifth generation (5G) applications, such as real-time streaming of ultra-high-definition (UHD) video and delivering augmented and virtual reality [1], [2]. This is partially driven by the abundant availability of mmWave spectrum in the unlicensed frequency bands that exist globally between 57 $66 \mathrm{GHz}$ [3]. Compared to competing microwave frequency bands, among the many favorable system attributes which can be attained (related to the shorter wavelength), include smaller antenna size, lower inter-cell interference [4] and the potential for improved security (e.g., preventing eavesdropping due to the higher propagation losses [5], [6]).

Although the use of mmWave frequencies may provide many benefits, such as those listed above, it will also equally present as many challenges. One of these relates to the increased susceptibility to blocking and shadowing caused by obstacles which reside in the local environment [7]. This is especially true for dense, indoor small cell deployments. For example, in [8] it was demonstrated that for a human body intersecting the line-of-sight (LOS) path of an indoor $60 \mathrm{GHz}$ point-to-point link, signal attenuations in the range of 20-40 dB can be induced. In [9] it was found that when a user moved from LOS to non-LOS (NLOS) relative to an indoor access point (AP) operating at $60 \mathrm{GHz}$, human body shadowing caused a $20 \mathrm{~dB}$ attenuation in the received signal power for both hallway and office environments. It was also discovered in [10] that when a mobile human intersected the mmWave LOS path, the received power could deteriorate by as much as $40 \mathrm{~dB}$. Similarly, it was observed in [11] that, compared to $60 \mathrm{GHz}$ wireless links with no obstructions, movements by multiple mobile users could cause sudden drops in the received signal of up to $40-50 \mathrm{~dB}$. Meanwhile, in [12], the wireless link between a $60 \mathrm{GHz}$ user equipment (UE) and a wall mounted AP was studied. It was observed that compared to LOS, under NLOS conditions, the received signal power can deteriorate by as much as $30 \mathrm{~dB}$ depending on the UE usage scenario.

One possible approach which can be used to mitigate signal deteriorations related to blocking and shadowing in mmWave small cell deployments is the use of a distributed antenna system (DAS) [13]. With this objective in mind, an indoor DAS system operating at $60 \mathrm{GHz}$ was investigated in [14]. Various switched diversity techniques were considered to assess their 


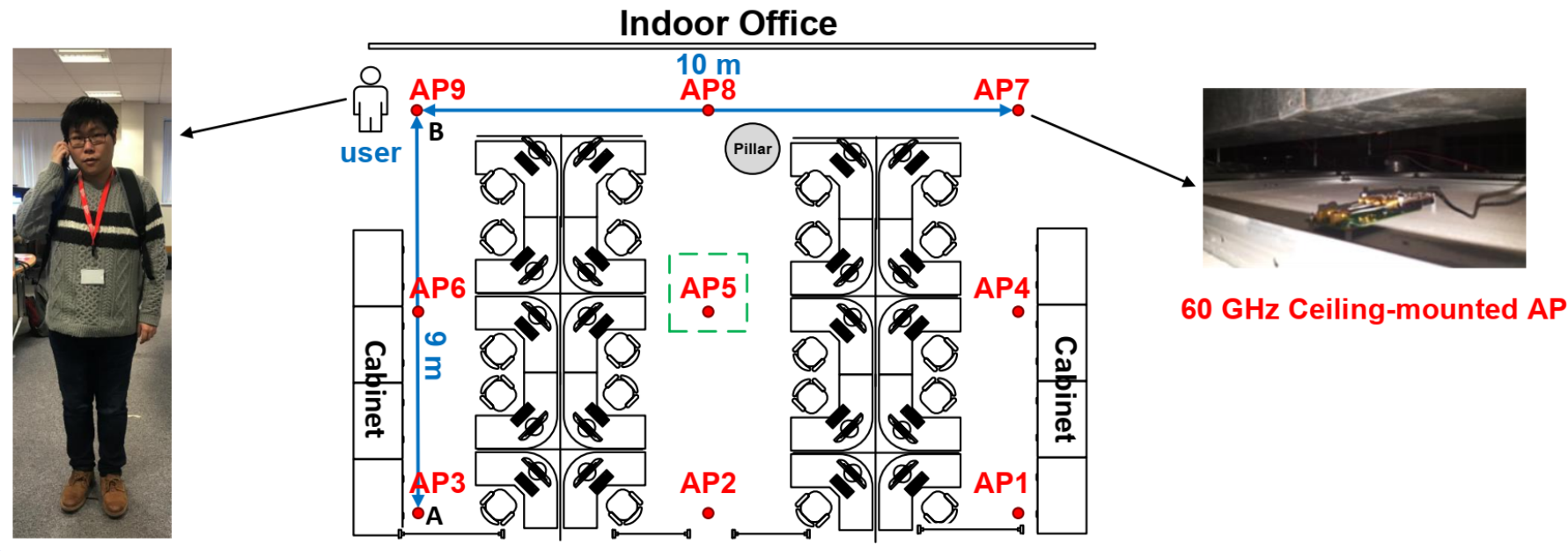

Fig. 1. Floor plan of the indoor mmWave measurement scenario environment along with a snapshot of the user imitating voice call (left) and an example $60 \mathrm{GHz}$ ceiling-mounted AP (right). It should be noted that AP5 (in the green dashed box) represents the target AP (Section III).

effectiveness for improving the overall performance. It was found in [14] that employing switch-and-examine combining can provide up to $9.1 \mathrm{~dB}$ of diversity gain. Nonetheless, the key metrics widely used in the analysis of diversitybased systems [15]-[17] and closely related to the diversity performance were omitted, namely the cross correlation [18] and the channel power imbalance (CPI) [19] between the signals observed at the different AP locations.

To the best of the author's knowledge, no empirically driven study of the cross correlation and CPI of the received signal strength (RSS) experienced in indoor DASs operating at $60 \mathrm{GHz}$ has appeared in the open literature. To address this issue and the shortcomings of [14], for the first time, we present the results of a series of experiments undertaken to characterize and provide new insights into the cross correlation and CPI encountered in indoor mmWave DASs. This has been achieved using a purposely developed measurement system which allowed simultaneous sampling of the received signal power at 9 ceiling mounted locations, using purposely developed $60 \mathrm{GHz}$ receivers (RXs). Using this setup, we have been able to compute the localized cross correlation coefficient (CCC) [20], [21] and CPI of the RSS across the mmWave DAS, while considering the signal transmitted from a mobile user who imitated making a voice call with a custom $60 \mathrm{GHz}$ transmitter (TX), emulating a UE. More importantly though, building upon a detailed statistical analysis of our results, we have been able to model the time evolution of the localized CCC and CPI using an autoregressive moving average (ARMA) model and an autoregressive integrated moving average (ARIMA) model, respectively. Subsequently, we reconstruct the empirical CCC and CPI time series via insample forecast. Furthermore, we discuss and compare the performances of different linear diversity combining techniques, i.e., selection combining (SC), equal-gain combining (EGC) and maximal-ratio combining (MRC), while also considering three AP selection mechanisms which will see deployment in future mmWave DASs, namely per-sample random AP selection [22], one-shot AP selection [23], and per-sample AP selection [23].

The remainder of this paper is organized as follows. The custom $60 \mathrm{GHz}$ measurement system and measurement scenarios are described in Section II. Section III discusses the cross correlation and CPI of the RSS, while the ARMA and ARIMA modeling approaches as well as related statistical tests are explained in Section IV. Additionally, in-sample forecast results obtained using the ARMA and ARIMA models are also provided. The localized diversity gain for different AP numbers as well as various AP selection mechanisms are presented and extensively discussed in Section V. Finally, the conclusions are summarized in Section VI.

\section{Measurement Set-Up and Scenarios}

\section{A. Measurement set-up}

To emulate a realistic indoor mmWave DAS, a custom $60 \mathrm{GHz}$ wireless channel measurement system was developed using the HMC6000LP711E TX ${ }^{1}$ and HMC6001LP711E RX ${ }^{2}$ modules manufactured by Analog Devices. Both units featured an identical linearly-polarized antenna-in-package with $+7.5 \mathrm{dBi}$ gain. Prior to the experiments, the antenna radiation pattern was measured in the anechoic chamber, located on the ground floor of the Institute of Electronics, Communications and Information Technology (ECIT) at Queen's University, Belfast, U.K. (further details can be found in [24]). The measured half power beam width (HPBW) of the antenna is approximately $120^{\circ}$ in both the azimuth and elevation ${ }^{3}$. To imitate a hypothetical $60 \mathrm{GHz} \mathrm{UE}$, the TX module was fixed to the inside of a compact acrylonitrile butadiene styrene (ABS) enclosure $(80 \mathrm{~mm} \times 80 \mathrm{~mm} \times 20 \mathrm{~mm})$ and configured to transmit a continuous wave signal centered at $60.05 \mathrm{GHz}$ with an Equivalent Isotropically Radiated Power (EIRP) of $+10.9 \mathrm{dBm}$.

\footnotetext{
${ }^{1}$ https://www.analog.com/media/en/technical-documentation/datasheets/hmc6000.pdf (visited on 13/11/2020)

${ }^{2}$ https://www.analog.com/media/en/technical-documentation/datasheets/hmc6001.pdf (visited on 13/11/2020)

${ }^{3}$ The measured antenna radiation patterns at $60 \mathrm{GHz}$ can be found in [24].
} 
The RX module was connected to a field programmable gate array (FPGA) based Red Pitaya data acquisition platform ${ }^{4}$ to emulate a mmWave AP (as seen on the right hand side of Figure 1). The $49 \mathrm{MHz}$ intermediate frequency (IF) signal output of the RX module was low pass filtered (with a $3 \mathrm{~dB}$ cutoff frequency at $50 \mathrm{MHz}$ ) to provide the function of antialiasing and then sampled using a 14-bit, 125 Msps analogto-digital converter (ADC) within the Red Pitaya platform. The FPGA unit was programmed to provide custom digital signal down conversion and the signal further filtered with a $3 \mathrm{~dB}$ bandwidth of $86.4 \mathrm{kHz}$. This implementation provided an effective channel sampling frequency of $96 \mathrm{kHz}$. The RSS was stored locally on the Red Pitaya platform and after the experiments was transferred to a desktop PC. During postprocessing, the RSS was downsampled by applying an averaging window of 48 consecutive samples to improve the signal to noise ratio (SNR) performance, resulting in an effective sampling rate of $2 \mathrm{kHz}$. Although not shown due to brevity, to verify the integrity of our collected RSS samples, we also conducted a study of the noise floor. Through our analysis, it was found that over $95 \%$ of the collected RSS data was between 15 and $60 \mathrm{~dB}$ greater than the noise floor for the paths $\mathrm{AB}$ and $\mathrm{BA}$, respectively. Additionally, the probability that a collected RSS sample was below the noise floor was evaluated and found to be less than $0.02 \%$.

\section{B. Measurement scenarios}

The measurements were conducted in an open office area $(10.62 \mathrm{~m} \times 12.23 \mathrm{~m})$, located on the 1st floor of the ECIT Institute at Queen's University Belfast, U.K. The floor plan is shown in Figure 1. The indoor office area consists of several metal studded dry walls with a metal tiled floor covered with polypropylene fiber, rubber backed carpet tiles, metal ceiling with mineral fiber tiles as well as recessed louvered luminaries suspended $2.70 \mathrm{~m}$ above the floor level. Also present are a number of metal cabinets, PCs, chairs, desks and soft partitions. During the measurements, $9 \mathrm{RX}$ boards were mounted on the ceiling at the points indicated by the red circles in Figure 1. These positions were chosen to emulate mmWave APs operating within a realistic indoor mmWave DAS. The RXs were positioned so that the antenna boresight was facing downwards, i.e., towards the floor level.

During the experiments, a test user (an adult male of height of $1.72 \mathrm{~m}$ and mass $75 \mathrm{~kg}$ ) imitated making a voice call, while holding the TX at his right ear. The TX antenna was oriented such that its boresight was facing outwards and away from the user's head. To ensure that any shadowing experienced in the experiments was the result of user-induced effects and signal obstruction within the test environment, the office area was unoccupied during the measurement process. Two trajectories were considered which involved the user walking along a straight line in two opposite directions, namely path $\mathrm{AB}$ and $\mathrm{BA}$, indicated using the blue line in Figure 1. The length of the path $\mathrm{AB}$ was $9 \mathrm{~m}$, while the average walking speed of the user was determined to be $1 \mathrm{~m} / \mathrm{s}$.

${ }^{4}$ https://pavel-demin.github.io/red-pitaya-notes/ (visited on 13/11/2020)

\section{Cross Correlation And Channel Power IMBALANCE}

After acquiring the raw RSS data, AP5 was selected as the target base station in the DAS since its overall mean was observed to be the highest for both paths $\mathrm{AB}$ and BA. Aiming to fully capture the temporal behavior of the CCCs between various AP pairs, the localized CCC, introduced in [20], was adopted in this work. This was obtained by computing the CCC using a moving window of length $N$ applied to the RSS data. An exploratory analysis of the data performed using the Kolmogorov-Smirnov (K-S) test indicated that the localized RSS observed at each of the APs was non-Gaussian in all cases. Consequently, the Spearman's rank-order correlation coefficient, was employed to calculate the localized CCCs between the RSS observed at different AP locations. The Spearman's rank-order correlation is a nonparametric measure of the monotonic relationship which exists between two variables in the ordinal form [25]. The localized CCCs were calculated as the sampled Spearman's rank-order correlation coefficient at the time instant $t$, denoted as $r_{S}(t)$, which can be written as

$$
r_{S}(t)=1-\frac{6 \sum_{i=1}^{N}\left[R_{X_{i j}}(t)-R_{X_{i k}}(t)\right]^{2}}{N\left(N^{2}-1\right)}, \quad(j \neq k)
$$

where $X_{i j}(t)$ and $X_{i k}(t)$ are the corresponding RSS values of the $i$ th sample at the time instant $t$, observed at the AP indexes of $j$ and $k$, respectively. In (1), $R_{X_{i j}}(t)$ and $R_{X_{i k}}(t)$ represent the ranks within the RSS vectors $X_{i j}(t)$ and $X_{i k}(t)$, respectively. In this study, three different values of $N$, (i.e., $400,1,000$ and 4, 000 , or equivalently $0.2 \mathrm{~s}, 0.5 \mathrm{~s}$ and $2 \mathrm{~s}$ ), were initially selected to investigate the impact of differing window sizes upon the CCC. For convenience, in the sequel, we refer to these three moving windows as short-term, medium-term and long-term, respectively.

As an example, the empirical probability density functions (PDFs) of the short, medium and long-term CCCs between AP5 and AP9 for paths AB and BA are presented in Figure 2. Also included in Figure 2 are theoretical plots for the best fitting Gaussian distributions. Compared to the mediumterm and long-term cases, it can be seen from Figures 2(a) and 2(d) that the Gaussian distribution provided a better fit for the short-term CCCs. This may be attributed to the fact that as $N$ increases, the number of wavelengths (distance) contained within the averaging window also increases and hence the signal propagation scenario can change significantly. The quantile-quantile (Q-Q) plots of the empirical short-term $r_{S}(t)$ and the corresponding Gaussian distribution fits are shown in Figures 3(a) and 3(b), respectively. Although slight fat-tailed (path $\mathrm{AB}$ ) and thin-tailed (path BA) artifacts can be observed in Figures 3(a) and 3(b), the majority of the empirical short-term $r_{S}(t)$ 's quantiles show a good match with those of the Gaussian fits. Therefore, $N$ was selected to be equal to 400 for all of the ensuing statistical analysis presented below.

Similar behavior to that described above, was also observed for other AP pairs. Therefore, the mean and standard deviation 

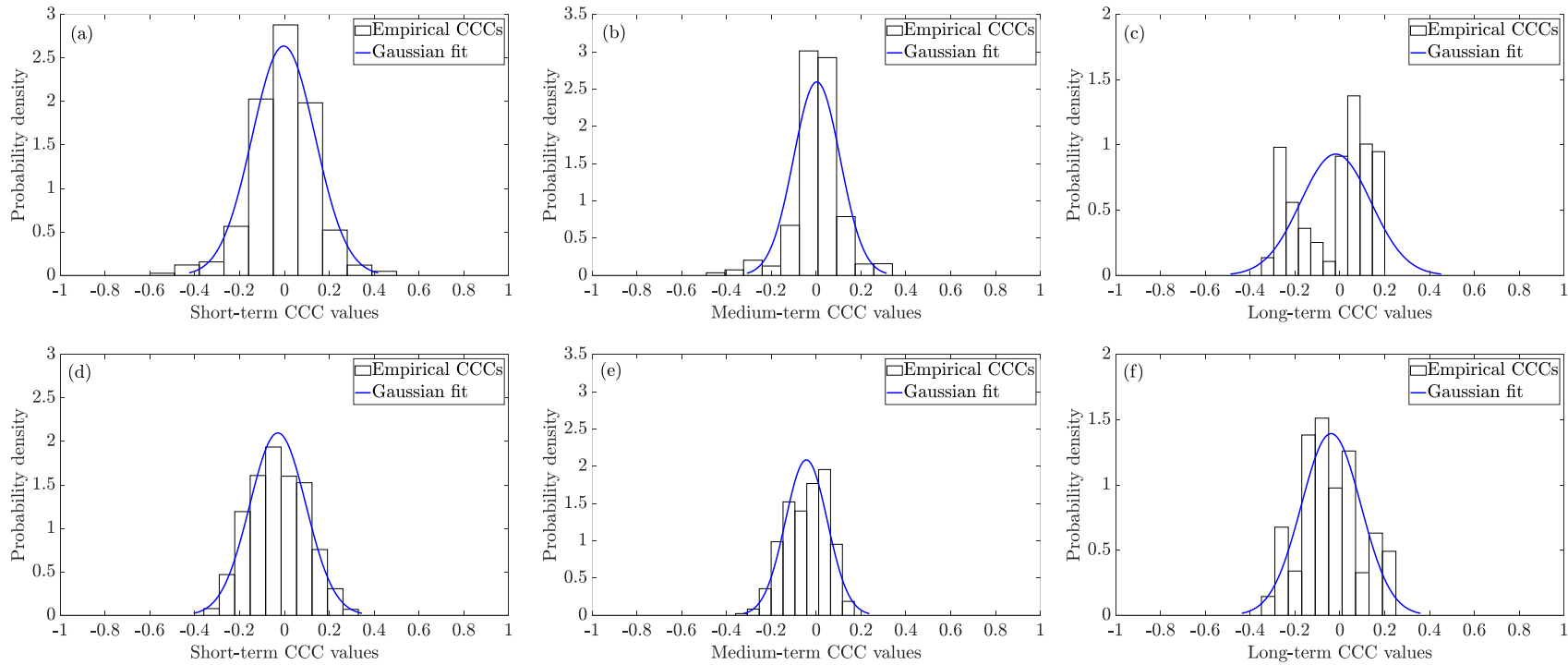

Fig. 2. Empirical PDFs of $r_{S}(t)$ between AP5 and AP9 along with the corresponding Gaussian distribution fits: (a) short-term $r_{S}(t)$ of the path AB, (b) medium-term $r_{S}(t)$ of the path $\mathrm{AB}$, (c) long-term $r_{S}(t)$ of the path $\mathrm{AB}$, (d) short-term $r_{S}(t)$ of the path $\mathrm{BA}$, (e) medium-term $r_{S}(t)$ of the path $\mathrm{BA}$, (f) long-term $r_{S}(t)$ of the path BA.
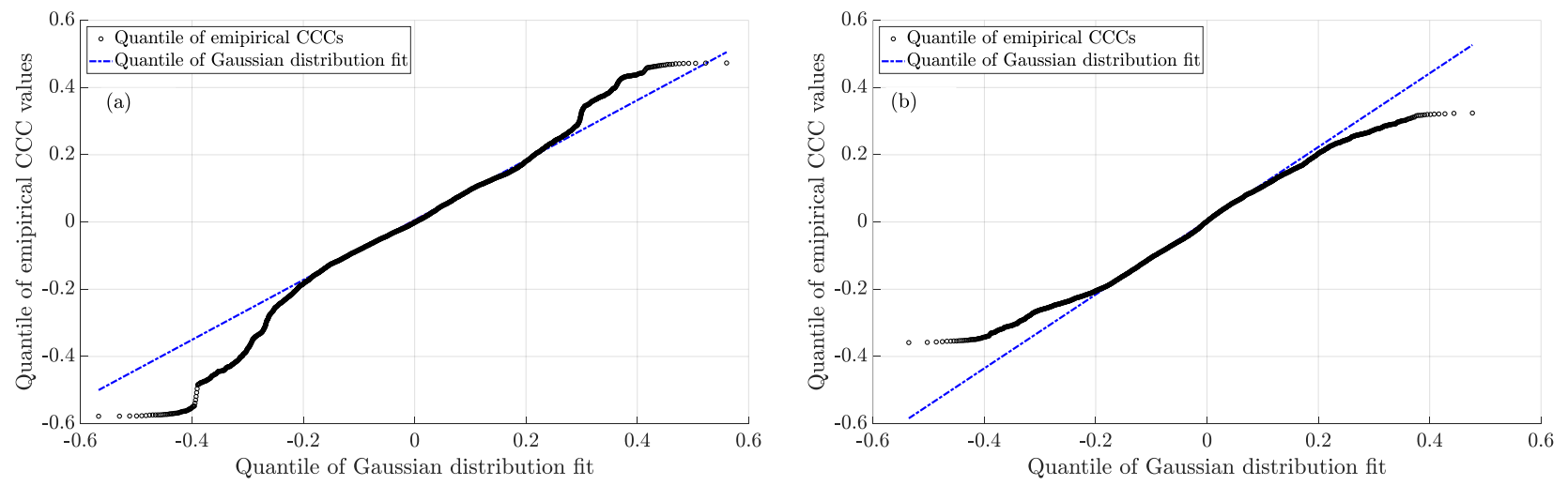

Fig. 3. Q-Q plots of short-term $r_{S}(t)$ and the corresponding Gaussian distribution fits between AP5 and AP9: (a) short-term $r_{S}(t)$ of the path AB, (b) short-term $r_{S}(t)$ of the path BA.

of $r_{S}(t)$, denoted as $\mu_{r_{S}}$ and $\sigma_{r_{S}}$, were obtained based on the corresponding best fitting Gaussian distributions. In the interest of brevity, the values of $\mu_{r_{S}}$ and $\sigma_{r_{S}}$ for all of the AP pairs are not shown. To summarize, it was found that $\mu_{r_{S}}$ ranged from -0.04 to 0.05 , while $\sigma_{r_{S}}$ was found to lie between 0.10 to 0.19 for all AP pairs. From these results, it can be concluded that the distribution of $r_{S}(t)$ can be approximated using a Gaussian distribution with $\mu=0$ and $\sigma$ ranging from 0.1 to 0.2 . When comparing the statistics of $r_{S}(t)$ between different AP pairs for both mobile paths, the $\mu_{r_{S}}$ and $\sigma_{r_{S}}$ values were typically found to be higher for the path $\mathrm{AB}\left(-0.04 \leq \mu_{r_{S}} \leq 0.05\right.$ and $\left.0.10 \leq \sigma_{r_{S}} \leq 0.19\right)$ than those of the path BA $\left(-0.04 \leq \mu_{r_{S}} \leq 0.03\right.$ and $0.10 \leq$ $\left.\sigma_{r_{S}} \leq 0.16\right)$. A possible reason for this is that with the user imitating making a voice call, the UE antenna boresight was facing outwards away from the user's head and towards the majority of the APs. As a result, the wireless links between the UE and APs were more likely to experience LOS/Quasi-LOS
(QLOS) conditions. Conversely, as shown in Figure 1, the UE antenna boresight was facing towards the metal cabinets for the majority of the path BA leading to QLOS/NLOS conditions between the UE and APs. Consequently, the observed CCC values between different AP pairs are generally higher with a wider spread because of the existence of LOS/QLOS paths, while under the NLOS conditions, the increased multipath tends to assist decorrelation and hence prompts lower CCC values with a smaller spread.

As with the computation of the localized CCC, the corresponding localized CPI at the time instant $t$ and denoted $C(t)$, can be expressed as

$$
\begin{aligned}
C(t) & =\left|20 \cdot \log _{10}\left(\frac{\sum_{i=1}^{N} X_{i j}(t) / N}{\sum_{i=1}^{N} X_{i k}(t) / N}\right)\right| \\
& =\left|20 \cdot \log _{10}\left(\frac{\sum_{i=1}^{N} X_{i j}(t)}{\sum_{i=1}^{N} X_{i k}(t)}\right)\right| .
\end{aligned}
$$


With $N=400$, the resultant PDFs of the localized CPI time series for different AP pairs were observed to be multimodal, thus the corresponding median of $C(t)$, denoted as $m_{C}$, was estimated, along with the minimum and maximum values of $C(t)$, denoted as $C_{\min }$ and $C_{\max }$. Comparing the statistics of $C(t)$ for the paths $\mathrm{AB}$ and $\mathrm{BA}$, the $m_{C}$ and $C_{\max }$ values were typically observed to be higher for the path AB. For instance, considering the pairing of AP5 and AP9, it was found that $m_{C}=7.0 \mathrm{~dB}, C_{\text {min }}=0.0 \mathrm{~dB}$ and $C_{\max }=22.6 \mathrm{~dB}$ for the path $\mathrm{AB}$, while $m_{C}=6.0 \mathrm{~dB}, C_{\min }=0.0 \mathrm{~dB}$ and $C_{\max }=13.6 \mathrm{~dB}$ for the path BA. This further verifies our observations for the channel conditions under both mobile paths. More significant power imbalance between the AP pairs can be observed for the path $\mathrm{AB}$ due to the existence of the LOS/QLOS links between the UE and the majority of APs. This situation means that the signal received at the APs is sensitive to the user location on the walk path and the relative orientation of the UE. This phenomenon appeared to have less of an influence for the path BA, as the increased multipath contribution tended to homogenize the signal received at the APs under QLOS/NLOS circumstances.

\section{ARMA AND ARIMA MODELING}

Having established some of the statistical properties of the key parameters associated with the performance of multiple antenna systems (i.e. CCC and CPI), in this section we investigate time series tools such as ARMA and ARIMA which can be used as a basis to model and simulate our empirical results. Prior to the model fitting, an augmented Dickey-Fuller (ADF) test [26] was used to check the stationarity properties of the localized CCC and CPI time series data. The $p$-values of the ADF test for the CCC time series obtained from the measurement data were found to be significantly less than $1 \%$, implying that the ADF test rejected the null hypothesis that a unit root existed in the univariate time series and thus we can conclude that the localized CCC time series data was stationary or trend-stationary. Turning our attention to the localized CPI time series, here, the $p$-values of the ADF test were observed to be notably higher than $5 \%$, suggesting that a unit root was present in the tested time series, and therefore the obtained localized CPI time series data was considered to be non-stationary. As a consequence, i.e. the stationarity of the localized CCC time series and non-stationarity of CPI time series, ARMA and ARIMA models were adopted to model and reconstruct the localized CCC and CPI time series, respectively.

Considering the mixture of an autoregressive (AR) process of $p$-th order, $\operatorname{AR}(p)$, and a moving average (MA) process of $q$-th order, $\operatorname{MA}(q)$, an ordinary $\operatorname{ARMA}(p, q)$ process [27] can be expressed as

$$
\left(1-\sum_{i=1}^{p} \phi_{i} L^{i}\right) x_{t}=\left(1+\sum_{i=1}^{q} \theta_{i} L^{i}\right) \varepsilon_{t}+c,
$$

where $L$ represents the lag operator, e.g., $x_{t-i}=L^{i} x_{t}$, and it can be seen from (3) that the output $x_{t}$ not only depends on the previous $p$ inputs of itself, but also relies on the previous $q$ inputs of a zero-mean Gaussian white noise process $\varepsilon_{t}$ (often referred to as residuals). In (3), $\left\{\phi_{1}, \phi_{2}, \ldots, \phi_{p}\right\}$ and $\left\{\theta_{1}, \theta_{2}, \ldots, \theta_{q}\right\}$ are coefficients that weight previous samples of the AR and MA processes, respectively. Additionally, $c$ represents a constant term, usually estimated as the mean value of $x_{t}$, i.e., $\mu_{x_{t}}$. The ARMA model is a suitable choice for the characterization of a wide-sense/weakly stationary stochastic process [27], but nevertheless, in real life some empirical time series data show evidence of non-stationarity. In order to model a stochastic process which exhibits non-stationarity, a more generalized model, referred to as an ARIMA model, is typically used. An $\operatorname{ARIMA}(p, d, q)$ process [27] can be defined as

$$
\left(1-\sum_{i=1}^{p} \phi_{i} L^{i}\right)(1-L)^{d} x_{t}=\left(1+\sum_{i=1}^{q} \theta_{i} L^{i}\right) \varepsilon_{t}+c,
$$

where $d$ is the degree of differencing. Comparing (3) with (4), the key difference between an ARMA and ARIMA process is the introduction of an additional differencing step $(d)$ which is applied ahead to eliminate the non-stationarity property within the time series data. Clearly, when $d=0$, an $\operatorname{ARIMA}(p, d, q)$ process naturally degenerates to an $\operatorname{ARMA}(p, q)$ one.

As discussed in the previous section, the values of $\mu_{r_{S}}$ were close to 0 for all the AP pairs, thus the value of $c$ was set to 0 for the ARMA model fits. Following the instructions in [28] and using the $\operatorname{ADF}$ test, an $\operatorname{ARIMA}(0, d, 0)$ process was initially applied to the localized CPI time series and it was found that the residuals of the time series' second-order differencing, i.e., $d=2$, were stationary or trend-stationary. Accordingly, $d$ was selected to be 2 for the ARIMA model fits presented afterwards. Moreover, the value of $c$ was also set to 0 for the ARIMA model since a constant is normally to be included when $d \leq 1$ but not for higher differencing orders [28].

There are many approaches available to select appropriate values for the orders $p$ and $q$. The maximum likelihood estimation (MLE) based second-order Akaike Information Criterion $\left(A I C_{C}\right)$ is often used due to its ability to take into account the trade-off between the goodness of fit and number of parameters used by the candidate model [29]. As shown in [30], the optimal model order can be determined by comparing the $A I C_{c}$ values of various order selections. Although the $A I C_{c}$ can effectively avoid the problem of overfitting [29], it does not always guarantee that the residuals obtained after ARMA or ARIMA modeling are in fact a white noise process. The residual's randomness can be examined by visually checking the autocorrelation function (ACF) and partial ACF (PACF) of the residuals against different confidence intervals (CIs) [31]. The $95 \%$ and $99 \%$ CIs can be approximated by $\pm 1.96 / \sqrt{n}$ and $\pm 2.576 / \sqrt{n}$ (here $n$ denotes the size of the test sample), respectively. An alternative approach to assess the randomness of the residuals is to apply the Ljung-Box test, which is calculated using the $Q$ statistic [32]. The $Q$ statistic of a test sample of size $n$ is defined as

$$
Q=n(n+2) \sum_{k=1}^{K} \frac{\hat{\rho}_{k}^{2}}{n-k}
$$



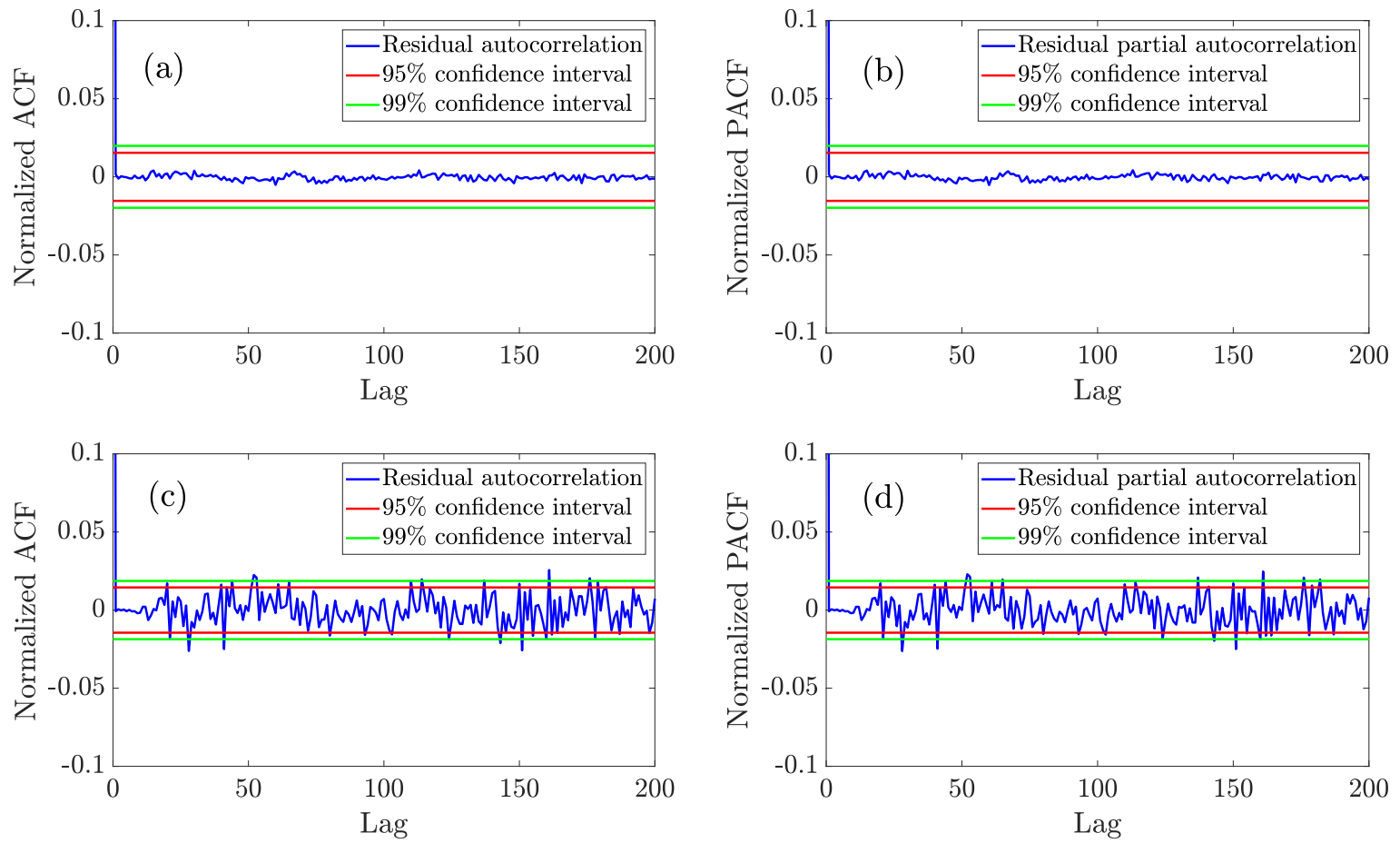

Fig. 4. Normalized ACF and PACF for the residuals of corresponding ARMA and ARIMA models for the short-term $r_{S}(t)$ and $C(t)$ between AP5 and AP9: (a) ACF of $\operatorname{ARMA}(9,1)$ residuals for the path $\mathrm{AB}$, (b) PACF of $\mathrm{ARMA}(9,1)$ residuals for the path $\mathrm{AB}$, (c) ACF of ARIMA(6, 2, 14) residuals for the path BA, (d) PACF of $\operatorname{ARIMA}(6,2,14)$ residuals for the path BA.

where $K$ is the number of lags to be tested and $\hat{\rho}_{k}$ is the sample autocorrelation at the corresponding lag of $k$. It is known from [32] that the $Q$ statistic asymptotically follows a chi-squared distribution with $K$ degrees of freedom, i.e., $\chi_{K}^{2}$, and under the ARMA/ARIMA residual test, the degrees of freedom should be adjusted with the orders of the corresponding model, e.g., in [33] it was suggested that for an $\operatorname{ARMA}(p$, $q)$ or an $\operatorname{ARIMA}(p, d, q)$ model, the degree of freedom should be modified to $K-p-q$. Therefore, in this work, we tested the residuals after ARMA and ARIMA model fitting with a $\chi^{2}$ distribution with $(K-p-q)$ degrees of freedom at both CIs of $95 \%$ and $99 \%$. After selecting the values of $p$ and $q$, the corresponding AR and MA coefficients were estimated based on the combination of conditional sum of squares and MLE (CSS-MLE) method, in which the speed and accuracy of the coefficients' computation can be optimally balanced [27].

It was found that the $A I C_{c}$ scores of the fitted ARMA model converged for the observed CCC time series across all the measurement scenarios when the order of the AR process was larger than 5 . Thus, different $\operatorname{ARMA}(p, q)$ models with $p \geq 5$ were investigated. It was observed that for the majority of the CCC time series obtained for the path $A B$, the residuals series of $\operatorname{ARMA}(9,1)$ passed the Ljung-Box test across all the required number of lags $K$ ranging from 20 to 40. Similarly, most of the CCC time series for the path BA were successfully modeled using an $\operatorname{ARMA}(5,1)$ process. Since there existed more APs experiencing LOS conditions for the path $\mathrm{AB}$ as opposed to path $\mathrm{BA}$, it is unsurprising that strong autocorrelation of the $\mathrm{CCCs}$ for path $\mathrm{AB}$ was noticed for a longer time lag, thus resulting in a larger AR order. It is noted that the residuals of a small number of AP pairs $(<10 \%$ overall) failed the Ljung-Box test at both CIs.

Now considering the collected CPI time series, the minimum $A I C_{c}$ scores of the fitted ARIMA model were obtained when the values of $p$ and $q$ did not exceed 10 and 20, respectively. Therefore, we explored ARIMA models with $p \leq 10$ and $q \leq 20$ for the localized CPI time series data. It was found that the $p$ and $q$ values of the optimal ARIMA model fit varied, depending on which AP pair was being considered. It is worth remarking that the residual time series of some AP pairs $(\approx 50 \%$ overall) failed the Ljung-Box test at both CIs across the number of lags $K$ ranging from 30 to 40 (recall that the degree of freedom for the Ljung-Box test, i.e., $K-p-q$, has to be positive). Moreover, it was observed that approximately $30 \%$ of the AP pairs failed the Ljung-Box test at the CI of $95 \%$ for most lags $K$ between 30 and 40 but passed at the CI of $99 \%$. To provide some further insights into the residual diagnostics, an example of the first 200-lag normalized ACF and PACF of the residuals obtained for the $r_{S}(t)$ and $C(t)$ between AP5 and AP9 are provided in Figure 4 . The corresponding estimated $95 \%$ and $99 \%$ CIs are also shown. Although the autocorrelations of a few time lags appear to be consequential, the normalized ACF and PACF of most time lags still lay within the CIs in Figure 4. Additionally, comparing the normalized ACF and PACF plots in Figures 4(a) and 4(b) with Figures 4(c) and 4(d), it is clear that the residuals 
TABLE I

ARMA COEFFICIENTS AND THE VARIANCE OF THE RESIDUALS FOR THE CCCS BETWEEN AP5 AND AP9 FOR PATHS AB AND BA

\begin{tabular}{c|c|c|c|c|c|c|c|c|c|c|c}
\hline \multirow{2}{*}{ Path } & \multicolumn{9}{|c|}{ ARMA Filter Coefficients } & \\
\cline { 2 - 12 } & $\phi_{1}$ & $\phi_{2}$ & $\phi_{3}$ & $\phi_{4}$ & $\phi_{5}$ & $\phi_{6}$ & $\phi_{7}$ & $\phi_{8}$ & $\phi_{9}$ & $\theta_{1}$ & $\operatorname{var}\left(\varepsilon_{t}\right)$ \\
\hline AB & 2.039 & -1.252 & 0.062 & 0.312 & -0.237 & 0.132 & -0.087 & 0.052 & -0.022 & -0.005 & $2.2 \times 10^{-5}$ \\
\hline BA & 2.870 & -3.339 & 2.075 & -0.720 & 0.114 & & & & - & -0.173 & $2.0 \times 10^{-6}$ \\
\hline
\end{tabular}
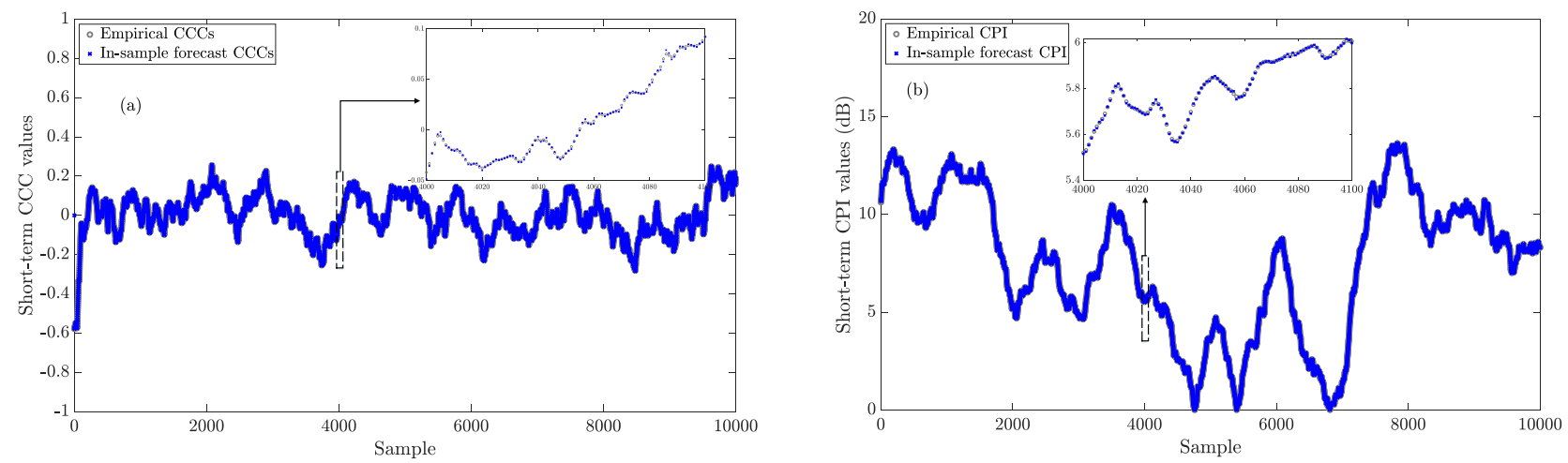

Fig. 5. Empirical and in-sample forecast CCC and CPI time series (first 10,000 samples) between AP5 and AP9 along with the expanded section between the sample index of 4,000 and 4,100: (a) CCCs of the path AB, (b) CPI of the path BA.

TABLE II

SELECTED ACCURACY MEASURES FOR THE ARMA AND ARIMA MODELS IN-SAMPLE FORECAST OF THE SHORT-TERM $r_{S}(t)$ AND $C(t)$ BETWEEN AP5 AND AP9 FOR PATHS AB AND BA

\begin{tabular}{c|c|c|c|c|c}
\hline & Path & MAE & RMSE & MPE & MASE \\
\hline \multirow{2}{*}{$r_{S}(t)$} & AB & $1.1 \times 10^{-3}$ & $4.7 \times 10^{-3}$ & $0.25 \%$ & 0.37 \\
\cline { 2 - 6 } & BA & $9.1 \times 10^{-4}$ & $1.5 \times 10^{-3}$ & $-0.07 \%$ & 0.39 \\
\hline \multirow{2}{*}{$C(t)$} & AB & $3.4 \times 10^{-3}$ & $4.5 \times 10^{-3}$ & $0.03 \%$ & 0.21 \\
\cline { 2 - 6 } & BA & $3.2 \times 10^{-3}$ & $4.1 \times 10^{-3}$ & $0.02 \%$ & 0.18 \\
\hline
\end{tabular}

after the ARIMA fitting exhibit stronger autocorrelations than those after the ARMA fitting. Hence more AP pairs were observed to fail the Ljung-Box test after the ARIMA modeling for the CPI time series. Nonetheless, the models still provided a very good fit to the data as demonstrated in the sequel.

Due to space limitations, we do not report the ARMA and ARIMA coefficients for the CCCs and CPI of all AP pairs. However, an example of the ARMA coefficients along with the variance of the residuals for the CCCs of AP5 and AP9 for both paths are presented in Table I. To further validate the ARMA and ARIMA model fitting, using the empirical $r_{S}(t)$ and $C(t)$ data as well as the corresponding ARMA and ARIMA coefficients as inputs, the fitted short-term $r_{S}(t)$ and $C(t)$ time series data, or so-called in-sample forecast time series, were reconstructed. An example of the first 10,000 empirical and in-sample forecast CCC and CPI time series data between AP5 and AP9 is presented in Figure 5. An expanded section between the sample indexes of 4,000 and 4,100 is also provided. From Figures 5(a) to 5(b), it can be seen that the insample forecast CCCs and CPI typically achieved an excellent match with the empirical CCCs and CPI (even if the residual series failed the Ljung-Box test at both CIs, such as the case in Figure 5(b)). Nevertheless, some slight forecast errors were still observed from the expanded section. Therefore, several commonly used accuracy measures were employed to quantify the in-sample forecast errors. The accuracy measures are now discussed below.

Let $h(t)$ and $\hat{h}(t)$ denote the empirical and in-sample forecast data at the time instant $t$, respectively, then the mean absolute error (MAE) can be defined as

$$
\text { MAE }=\frac{\sum_{t=1}^{n}|h(t)-\hat{h}(t)|}{n},
$$

where $n$ represents the sample number of the empirical and in-sample forecast data.

The root-mean-square error (RMSE) between the empirical $h(t)$ and in-sample forecast $\hat{h}(t)$ can be defined as

$$
\mathrm{RMSE}=\sqrt{\frac{\sum_{t=1}^{n}[h(t)-\hat{h}(t)]^{2}}{n}} .
$$

The mean percentage error (MPE) is defined as the computed average of percentage errors by the forecast samples of a model differing from observed values, and can be expressed as

$$
\text { MPE }=\frac{100 \%}{n} \sum_{t=1}^{n} \frac{[h(t)-\hat{h}(t)]}{h(t)} .
$$

The mean absolute scaled error (MASE), which is determined by the MAE of the forecast divided by the MAE of the in-sample one-step naive forecast [34], is given as

$$
\text { MASE }=\frac{n-1}{n} \frac{\sum_{t=1}^{n}|h(t)-\hat{h}(t)|}{\sum_{t=2}^{n}|h(t)-h(t-1)|},
$$

a MASE value of larger than one implies that the actual insample forecast performs worse than a simple naive forecast, 

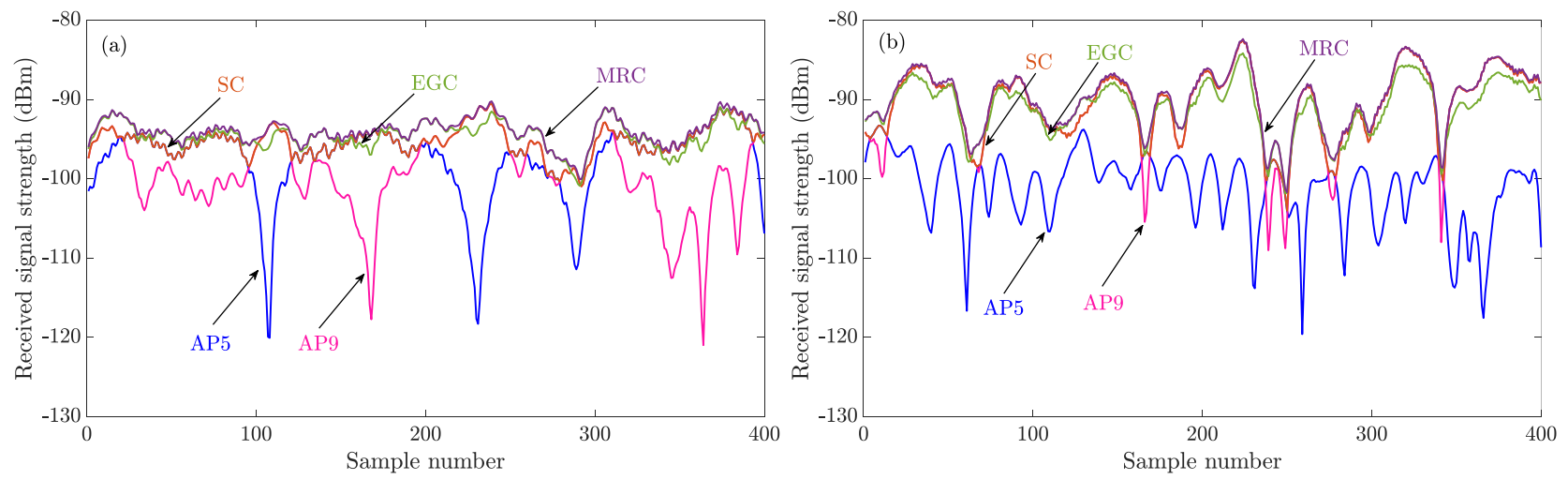

Fig. 6. The RSS of the first 400 samples (equivalently 0.2 s) at AP5 and AP9 along with the SC output, EGC output, and MRC output: (a) path AB, (b) path BA.

while a value of MASE less than one indicates that the insample forecast outperforms the naive forecast. The selected accuracy measures for the ARMA and ARIMA models insample forecast of the short-term $r_{S}(t)$ and $C(t)$ between AP5 and AP9 for both mobile paths are presented in Table II. It can be clearly seen from Table II that compared to the empirical observations for $r_{S}(t)$ and $C(t)$, the in-sample forecast achieved a very high accuracy, e.g., in the case of the $C(t)$ time series estimation, the MPE of the paths $\mathrm{AB}$ and BA was only $0.03 \%$ and $0.02 \%$, respectively. Furthermore, the MASE of the in-sample forecast of $r_{S}(t)$ was 0.37 and 0.39 for the paths $\mathrm{AB}$ and $\mathrm{BA}$, indicating that the $\operatorname{ARMA}(9$, $1)$ and $\operatorname{ARMA}(5,1)$ models provided a much better in-sample forecasting performance than the naive forecast, thus verifying the feasibility of our ARMA modeling approach.

\section{Diversity Gain}

Three types of diversity combining technique, namely SC, EGC, and MRC, were considered to combine the signal received at the APs forming the mmWave DAS. SC is a switched combining technique that selects the AP with the highest RSS at each sample interval [35]. For an $M$-AP DAS which utilizes SC, the signal envelope at the output, $L_{\mathrm{SC}}(i)$, can be written as

$$
L_{\mathrm{SC}}(i)=\max \left(X_{1}(i), X_{2}(i), \cdots, X_{M}(i)\right),
$$

where $X_{M}(i)$ is the signal envelope of the $i$ th sample at the $M$ th AP of the DAS.

EGC considers the case where the channel gains of different APs are equal. Following this, an equal-gain combiner simply assigns an equal weight to the signal envelope observed at each AP [35], thus the instantaneous sample output of an $M$-AP EGC DAS can be written as

$$
L_{\mathrm{EGC}}(i)=\frac{X_{1}(i)+X_{2}(i)+\cdots+X_{M}(i)}{\sqrt{M}} .
$$

Unlike the EGC approach, MRC is performed by weighting the sampled signal of each AP with respect to its own instantaneous SNR. If we assume the noise is uncorrelated with the received signal at each AP location and the noise power is equal to unity for all the utilized APs, the instantaneous sample output of an $M$-AP MRC DAS can be expressed as

$$
L_{\mathrm{MRC}}(i)=\sqrt{X_{1}^{2}(i)+X_{2}^{2}(i)+\cdots+X_{M}^{2}(i)} .
$$

An important performance metric in the context of diversity combining is diversity gain. For a macro diversity system such as a DAS, it is defined as the difference between the signal level of a target AP and that received at the output of the DAS for a given signal reliability [36]. Following the studies conducted in [18] and [36], in this work the diversity gain was evaluated at a signal reliability of $90 \%$. Similar to the computation of the localized CCC and CPI, we computed the localized diversity gain by considering a moving window of length $N$ applied to the recorded data. Within each window, we combined the raw RSS from the relevant APs on a sample by sample basis using the appropriate combining scheme. For each realization of the moving window, we then calculated the diversity gain as described above. This process was repeated as the window of length $N$ was moved across the recorded data to obtain the localized diversity gain time series. To keep our analysis of the localized diversity gain consistent with that for the localized CCC and CPI, $N$ was set equal to 400 .

To visually check the improvement associated with the use of each of the combining techniques, an example of the output from a dual-AP DAS (AP5 and AP9), considering the first 400 samples, is shown in Figure 6. It can be seen from Figure 6(a) and Figure 6(b) that, for both mobile paths, when one of the APs suffered from deep fading caused by shadowing and blocking, the other AP typically experienced better channel conditions thus offering the opportunity to exploit the spatial diversity offered by the mmWave DAS. The corresponding empirical cumulative distribution functions (CDFs), which illustrate the improvement in the signal reliability achieved using a dual-AP indoor DAS, are provided in Figure 7. It can be seen from Figure 7(a) and Figure 7(b) that, compared to using only the target AP, the dual-AP DAS significantly improved the received signal reliability performance for both paths, e.g., the localized EGC gains were $6.7 \mathrm{~dB}$ and $11.3 \mathrm{~dB}$ 

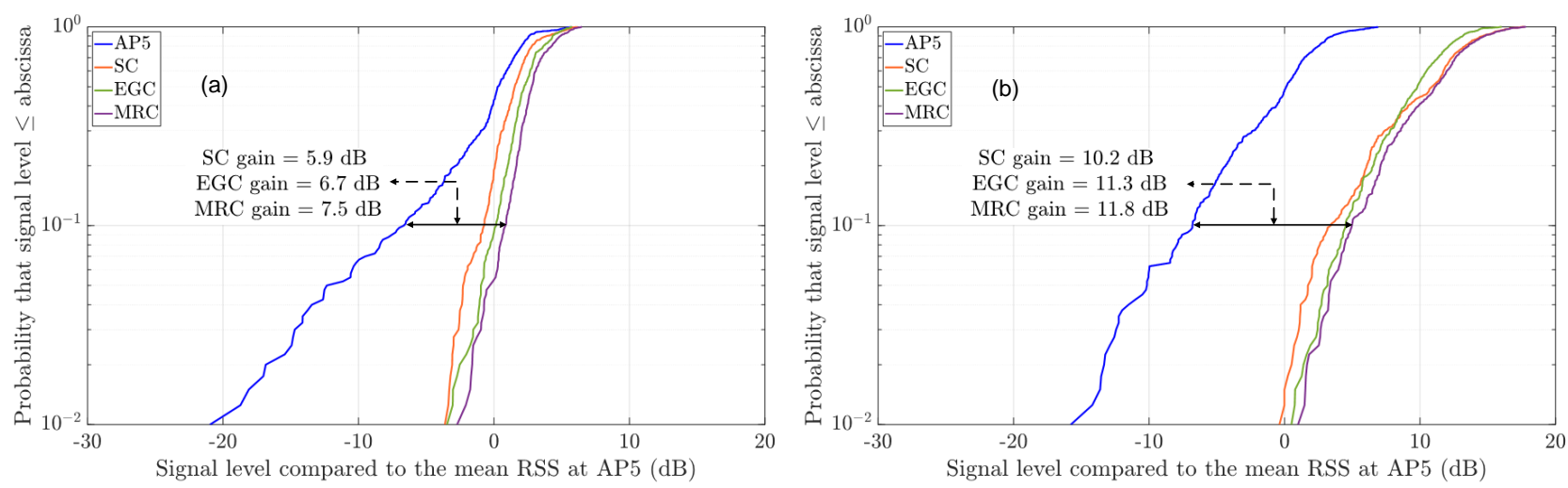

Fig. 7. Empirical CDFs of the $60 \mathrm{GHz}$ indoor macro diversity system considering the first 400 samples (equivalently $0.2 \mathrm{~s}$ ) of the RSS at AP5 and AP9: (a) path $\mathrm{AB}$, (b) path BA. Note that all CDFs are relative to the mean of the first 400 samples of the RSS at AP5.

for the paths $\mathrm{AB}$ and $\mathrm{BA}$, respectively. It is also interesting to note that for the first 400 samples (equivalently $0.2 \mathrm{~s}$ ), the values of the localized diversity gains for path BA were found to be larger than those for path $\mathrm{AB}$, e.g., the localized $\mathrm{SC}$ gain was $10.2 \mathrm{~dB}$ for the path $\mathrm{BA}$, while the localized $\mathrm{SC}$ gain was only $5.9 \mathrm{~dB}$ for the path $\mathrm{AB}$. A possible reason for this can be deduced from Figure 1. More precisely, when the user started moving along the path BA, the distance between the UE and AP9 was much smaller than that between the UE and AP5, meaning that the RSS values at AP9 were generally larger than those at AP5 (see Figure 7(b)).

Even though it has been demonstrated that simply using a dual-AP DAS can lead to considerable improvements in the signal reliability, it is still useful to explore the potential for further enhancements that can be achieved by using an even greater number of APs. This is a typical consideration often associated with macro diversity systems, and is commonly referred to as the base station (BS) or AP selection problem [37], [38]. It is especially important at mmWave frequencies since a dense deployment of APs is desirable to mitigate the effects of high path loss and possible LOS blockages. Another factor which must be taken into account when considering macro diversity systems such as DAS relates to the additional signal processing which must be performed to combine the information received at multiple APs. This typically requires additional hardware and the burden of handling coordination between APs, which can lead to increased power consumption [39]. In the sequel, we consider three different AP selection mechanisms which have been proposed for use in future mmWave DAS deployments [22], [23]. We compare their performance in terms of the diversity gain that can be achieved when considering different numbers of APs. The AP selection mechanisms are now listed as follows:

- Per-sample random AP selection [22]: As the name suggests, an AP is selected randomly at each sample interval. This is the simplest AP selection mechanism because the random AP selection does not require any RSS information. For a fixed number of APs $(M)$, the mean localized per-sample random AP selection diversity gain, denoted as $G_{\mathrm{RS}}$, can be written as

$$
G_{\mathrm{RS}}=\frac{1}{U T} \sum_{u=1}^{U} \sum_{t=1}^{T} G(u, t),
$$

where $U$ represents the number of possible AP pairs for a fixed value of $M$, and $U$ can be calculated as $C_{M}^{O}=\left(\begin{array}{l}O \\ M\end{array}\right)$ (here $C$ is the combination operator and $O$ denotes the maximum number of APs). Besides, $T$ represents the total sample number of the collected localized diversity gain time series, i.e., $G(u, t)$.

- One-shot AP selection [23]: One-shot AP selection is achieved by persisting with the connections established between the UE and APs in the first time slot. During the first time slot, the UE simply selects the AP pairs that can achieve the maximum localized diversity gain. This means the UE will communicate with the same APs for the remaining time slots and can not choose other APs. This AP selection mechanism is more complex than the per-sample random AP selection in terms of the signal processing since a computation of $C_{M}^{O}$ is required to select the one-shot AP pairs. The mean localized one-shot AP selection gain, denoted as $G_{\mathrm{OS}}$, can be expressed as

$$
G_{\mathrm{OS}}=\frac{1}{T} \sum_{t=1}^{T} \underset{\substack{u \in\left[1, C_{M}^{O}\right] \\ t=1}}{\arg \max } G(u, t) .
$$

- Per-sample optimal AP selection [23]: This is the most complex and optimal approach. Under this scheme, optimal matching between the UE and APs is determined by choosing the AP pairs that achieve the maximum localized diversity gain in each time sample. Under this scheme, it is entirely possible that the UE could connect to different AP pairs in each sample interval. An overall computation of $C_{M}^{O} \cdot T$ is needed in order to obtain the per-sample optimal gain performance. The mean localized per-sample optimal AP selection gain, denoted as $G_{\mathrm{OPT}}$, can be written as

$$
G_{\mathrm{OPT}}=\frac{1}{T} \sum_{t=1}^{T} \underset{\substack{u \in\left[1, C_{M}^{O}\right] \\ t=i}}{\arg \max } G(u, t),(1 \leq i \leq T) .
$$



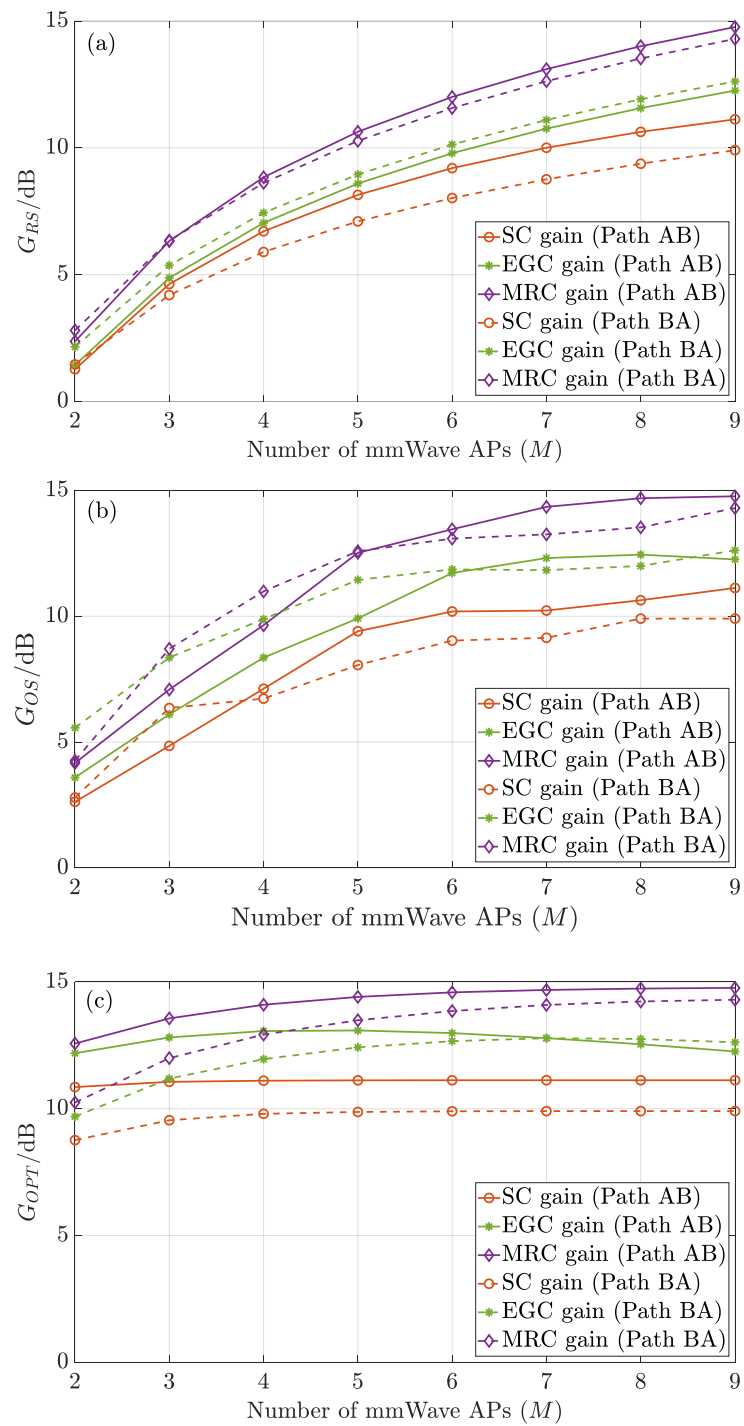

Fig. 8. The impact of using increasing numbers of mmWave APs on the mean localized diversity gain for the path $\mathrm{AB}$ (solid) and BA (dashed): (a) per-sample random AP selection, (b) one-shot AP selection, (c) per-sample optimal AP selection.

In this work, the value of $O$ was 9 , since 9 mmWave APs were deployed in the DAS. Correspondingly, $M$ was varied from 2 to 9 . Additionally, the values of $T$ were set to be 16891 and 18079 (i.e. the total number of samples within the collected localized diversity gain time series) for the paths $\mathrm{AB}$ and BA, respectively. The impact of using increasing numbers of mmWave APs and different AP selection mechanisms on the mean localized diversity gain for the paths $\mathrm{AB}$ and $\mathrm{BA}$ are shown in Figures 8(a) to 8(c). As expected, for a fixed number of mmWave APs, the per-sample random AP selection always performed worst, while the per-sample optimal AP selection provided the best performance for both of the considered mobile paths, e.g., when $M=5$, for the path $\mathrm{AB}$, the value of $\mathrm{SC}$ $G_{\mathrm{RS}}$ was only $8.1 \mathrm{~dB}$ (Figure $8(\mathrm{a})$ ), while the corresponding values of the SC $G_{\mathrm{OS}}$ and $G_{\mathrm{OPT}}$ were $9.4 \mathrm{~dB}$ and $11.1 \mathrm{~dB}$ (Figure 8(b) and Figure 8(c)), respectively. This observation is the result of the per-sample random AP selection simply choosing AP pairs randomly while ignoring the instantaneous feedback contained within the RSS information. Conversely, the per-sample optimal AP selection updates the AP selection by choosing the AP pairs with the maximum localized gain per sample interval.

Furthermore, for the one-shot and per-sample optimal AP selection mechanisms, the diversity gain improvements were observed to 'saturate' with the increasing numbers of mmWave APs. For instance, for the one-shot AP selection mechanism in Figure $8(\mathrm{~b})$, when $M$ is increased from 3 to 6 , the EGC $G_{\mathrm{OS}}$ for the path $\mathrm{AB}$ increased from $6.1 \mathrm{~dB}$ to $11.7 \mathrm{~dB}$. Meanwhile, when $M$ was further increased from 6 to 9 , the EGC $G_{\mathrm{OS}}$ for the path $\mathrm{AB}$ only slightly increased from $11.7 \mathrm{~dB}$ to $12.3 \mathrm{~dB}$. A similar phenomenon was also observed in the per-sample optimal AP selection mechanism (Figure 8(c)). This was due to the fact that with an increasing number of mmWave APs, values of the localized CCC and CPI between different AP locations also unavoidably increase, thus providing a ceiling for the DAS diversity gain improvements [18].

Most importantly, from the mean localized diversity gain plots provided in Figure 8, we can identify the optimal number of APs for each selection mechanism. For instance, for the per-sample random AP selection in Figure 8(a), it can be observed that up until $M=6$, significant improvements in the diversity gain are achieved for all the combining schemes. Although after this point, improvements in the diversity gain were not as significant as subsequent APs were added to the system. Similar to the per-sample random AP selection, the greatest improvements in the diversity gain for the one-shot AP selection were observed for successive additions of the APs until $M=6$ (Figure $8(\mathrm{~b})$ ). After this, the value of $G_{\text {OS }}$ only marginally increased for both mobile paths, e.g., when $M$ increased from 6 to 9 , the MRC $G_{\mathrm{OS}}$ for the path $\mathrm{AB}$ only increased from $13.5 \mathrm{~dB}$ to $14.8 \mathrm{~dB}$ (i.e., a $1.3 \mathrm{~dB}$ improvement). Considering the extra system complexities introduced by including additional mmWave APs, using 6 APs appears to provide the optimal diversity gain performance for the one-shot AP selection mechanism. Compared to the previous two AP selection mechanisms, as shown in Figure $8(\mathrm{c})$, the $G_{\text {OPT }}$ converged to its optimal values at a much faster rate for both paths $\mathrm{AB}$ and $\mathrm{BA}$. This behavior is particularly noticeable for the SC and MRC schemes for $M>4$. The reason for this is that the per-sample optimal AP selection evaluates the connections among different AP pairs during the sample interval, thus the same favorable AP pairs will always be chosen within a specific time and increasing the number of mmWave APs simply adds additional 'noisy' APs to the previously selected 'optimal' AP pairs. This can be further verified for the EGC technique when $M$ was larger than 4 . Here the values of $G_{\text {OPT }}$ were discovered to actually decrease since EGC assigns the same weight to each of the APs regardless of the individual link's quality, e.g., when $M$ increased from 4 to 9 , the values of $G_{\mathrm{OPT}}$ for the path $\mathrm{AB}$ decreased from $13.1 \mathrm{~dB}$ to $12.3 \mathrm{~dB}$. When considering the tradeoffs encountered between processing overhead and achievable diversity gain, EGC appears to offer the best overall performance. Not only did the EGC scheme perform better than the SC scheme (see Figure 8(a) to Figure $8(\mathrm{c})$ ), it 
also requires less processing overhead compared to the MRC scheme (due to the fact that in MRC, the weight assigned to each AP has to be constantly updated by monitoring the simultaneous RSS observed at various AP locations [35]).

\section{CONCLUSION AND Future WORK}

Prior to the successful deployment of DASs, it is necessary to understand many of the factors related to signal propagation which can affect system performance. To this end, we have empirically studied the signal received from a hypothetical UE operating at $60 \mathrm{GHz}$ in an indoor environment by 9 ceilingmounted APs. In particular, we characterized a number of the key metrics governing the performance of DASs such as the cross correlation, channel power imbalance and diversity gain using a range of time series tools. Our results have shown that the distribution of the CCCs between various AP pairs was well described by a Gaussian distribution with $\mu=0$ and $\sigma$ varying between 0.1 and 0.2 . Additionally, when compared to NLOS scenarios, the existence of LOS and QLOS signal paths produced higher CCCs values with increased spread.

Applying ARMA models to the CCC time series, it was found that processes of order $\operatorname{ARMA}(9,1)$ and $\operatorname{ARMA}(5$, 1) could be used to satisfactorily model the evolution of the $\mathrm{CCC}$ for paths $\mathrm{AB}$ and $\mathrm{BA}$ (representing two opposite mobile directions), respectively. Similar to the CCC, the presence of LOS and QLOS signal paths induced higher CPI values. As the CPI typically displayed non-stationary statistical characteristics, it was necessary to model the CPI process using an ARIMA model. For this purpose, it was found that the CPI time series could be adequately modeled and predicted using an $\operatorname{ARIMA}(p, 2, d)$ process, with $p \leq 10$ and $q \leq 20$. The validity of the ARMA and ARIMA models was examined by visually checking the $\mathrm{ACF}$ and PACF of the acquired residual series, using the Ljung-Box test as well as a comparison of the CCC and CPI time series in-sample forecast and those of the experimental results. Despite failing the Ljung-Box test for $\approx 50 \%$ of the considered cases, the resultant normalized ACF and PACF across most time lags was observed to fall within the CIs of $95 \%$ and $99 \%$. Furthermore, the in-sample forecast (and associated error measures) have shown that ARIMA models can still be used to provide an adequate prediction of the CPI time series evolution.

Lastly, we considered three diversity combining techniques (SC, EGC and MRC) along with three AP selection mechanisms for use in mmWave DAS. It was found that the persample optimal AP selection provided the highest diversity gains albeit at the cost of additional computations. Most notably, based on the diversity gain results, it was ascertained that utilizing greater numbers of mmWave APs generally provided a better performance for the per-sample random AP selection mechanism, while the performance improvements beyond a certain number of mmWave APs became restricted for the one-shot and per-sample optimal AP selection mechanisms. It was shown that a 6-AP with one-shot AP selection and 4-AP with per-sample optimal AP selection mmWave DAS should provide satisfactory diversity gain improvements. Additionally, the EGC scheme was observed to provide a better performance than the SC scheme, with less complexity compared to the MRC scheme.

Although some promising results have been obtained for the use of mmWave DASs, several open research topics remain which should be explored in the future work. For example, in this work we considered a single mobile user imitating a voice call application within an unoccupied indoor office environment. In the future, the measurement scenarios could be extended to consider other daily UE use cases, e.g., using a messaging application or simply considering when the UE is carried in a pocket or bag. Further investigations should also take into account other factors which could affect the system performance such as the presence of other people within the indoor environment. Other switched combining techniques, e.g., switch-and-stay and switch-and-examine combining [36], [40], should be explored and the associated performance metrics analyzed for future mmWave DAS deployments.

\section{REFERENCES}

[1] M. Xiao, S. Mumtaz, Y. Huang, L. Dai, Y. Li, M. Matthaiou, G. K. Karagiannidis, E. Björnson, K. Yang, I. Chih-Lin et al., "Millimeter wave communications for future mobile networks," IEEE Journal on Selected Areas in Communications, vol. 35, no. 9, pp. 1909-1935, 2017.

[2] X. Ge, S. Tu, G. Mao, C.-X. Wang, and T. Han, "5G ultra-dense cellular networks," IEEE Wireless Communications, vol. 23, no. 1, pp. 72-79, 2016.

[3] C. Park and T. S. Rappaport, "Short-range wireless communications for next-generation networks: UWB, $60 \mathrm{GHz}$ millimeter-wave WPAN, and ZigBee," IEEE Wireless Communications, vol. 14, no. 4, 2007.

[4] C.-X. Wang, J. Bian, J. Sun, W. Zhang, and M. Zhang, "A survey of $5 \mathrm{G}$ channel measurements and models," IEEE Communications Surveys \& Tutorials, vol. 20, no. 4, pp. 3142-3168, 2018

[5] L. Wei, R. Q. Hu, Y. Qian, and G. Wu, "Key elements to enable millimeter wave communications for $5 \mathrm{G}$ wireless systems," IEEE Wireless Communications, vol. 21, no. 6, pp. 136-143, 2014.

[6] P. Wang, Y. Li, L. Song, and B. Vucetic, "Multi-gigabit millimeter wave wireless communications for 5G: From fixed access to cellular networks," IEEE Communications Magazine, vol. 53, no. 1, pp. 168$178,2015$.

[7] P. Karadimas, B. Allen, and P. Smith, "Human body shadowing characterization for $60-\mathrm{GHz}$ indoor short-range wireless links," IEEE Antennas and Wireless Propagation Letters, vol. 12, pp. 1650-1653, 2013.

[8] S. Collonge, G. Zaharia, and G. E. Zein, "Influence of the human activity on wide-band characteristics of the $60 \mathrm{GHz}$ indoor radio channel," IEEE Transactions on Wireless Communications, vol. 3, no. 6, pp. 2396-2406, 2004.

[9] S. K. Yoo, S. L. Cotton, Y. J. Chun, W. G. Scanlon, and G. A. Conway, "Channel characteristics of dynamic off-body communications at $60 \mathrm{GHz}$ under line-of-sight (LOS) and non-LOS conditions," IEEE Antennas and Wireless Propagation Letters, vol. 16, pp. 1553-1556, 2017.

[10] T. S. Rappaport, G. R. MacCartney, S. Sun, H. Yan, and S. Deng, "Small-scale, local area, and transitional millimeter wave propagation for 5G communications," IEEE Transactions on Antennas and Propagation, vol. 65, no. 12, pp. 6474-6490, 2017.

[11] B. Peng, S. Rey, D. M. Rose, S. Hahn, and T. Kuerner, "Statistical characteristics study of human blockage effect in future indoor millimeter and sub-millimeter wave wireless communications," in 2018 IEEE 87th Vehicular Technology Conference (VTC Spring). IEEE, 2018, pp. 1-5.

[12] S. K. Yoo, S. L. Cotton, R. W. Heath, and Y. J. Chun, "Measurements of the $60 \mathrm{GHz}$ UE to eNB channel for small cell deployments," IEEE Wireless Communications Letters, vol. 6, no. 2, pp. 178-181, 2017.

[13] A. A. Saleh, A. Rustako, and R. Roman, "Distributed antennas for indoor radio communications," IEEE Transactions on Communications, vol. 35, no. 12 , pp. $1245-1251,1987$.

[14] S. K. Yoo, L. Zhang, S. L. Cotton, and H. Q. Ngo, "Distributed antenna systems used for indoor UE to access point communications at $60 \mathrm{GHz}$," in 2019 13th European Conference on Antennas and Propagation (EuCAP), 2019, pp. 1-5. 
[15] K. Zayana and B. Guisnet, "Measurements and modelisation of shadowing cross-correlations between two base-stations," in ICUPC'98. IEEE 1998 International Conference on Universal Personal Communications. Conference Proceedings (Cat. No. 98TH8384), vol. 1, 1998, pp. 101105.

[16] B. Yanakiev, J. Ø. Nielsen, M. Christensen, and G. F. Pedersen, "On small terminal antenna correlation and impact on MIMO channel capacity," IEEE Transactions on Antennas and Propagation, vol. 60, no. 2, pp. 689-699, 2011.

[17] I. Khan, P. S. Hall, A. A. Serra, A. R. Guraliuc, and P. Nepa, "Diversity performance analysis for on-body communication channels at 2.45 GHz," IEEE Transactions on Antennas and propagation, vol. 57, no. 4, pp. 956-963, 2009.

[18] A. M. Turkmani, A. A. Arowojolu, P. Jefford, and C. Kellett, "An experimental evaluation of the performance of two-branch space and polarization diversity schemes at $1800 \mathrm{MHz}$," IEEE Transactions on Vehicular Technology, vol. 44, no. 2, pp. 318-326, 1995.

[19] B. B. Haile, A. A. Dowhuszko, J. Hämäläinen, R. Wichman, and Z. Ding, "On performance loss of some CoMP techniques under channel power imbalance and limited feedback," IEEE Transactions on Wireless Communications, vol. 14, no. 8, pp. 4469-4481, 2015.

[20] L. J. November, "Measurement of geometric distortion in a turbulent atmosphere," Applied Optics, vol. 25, no. 3, pp. 392-397, 1986.

[21] N. Bhargav, S. L. Cotton, and V. F. Fusco, "Signal reception characteristics in the proximity of Alice and Bob for secure indoor peer-to-peer communications at $2.45 \mathrm{GHz}$," in 2015 9th European Conference on Antennas and Propagation (EuCAP), 2015, pp. 1-4.

[22] C. Skouroumounis, C. Psomas, and I. Krikidis, "Low-complexity base station selection scheme in mmWave cellular networks," IEEE Transactions on Communications, vol. 65, no. 9, pp. 4049-4064, 2017.

[23] X. Qin, X. Yuan, Z. Zhang, F. Tian, T. Hou, and W. Lou, "Joint user-AP association and resource allocation in multi-AP $60 \mathrm{GHz}$ WLAN," IEEE Transactions on Vehicular Technology, vol. 68, no. 6, pp. 5696-5710, 2019.

[24] L. Zhang, A. McKernan, S. L. Cotton, and W. G. Scanlon, "The influence of elevation angle on $60 \mathrm{GHz}$ near-body path gain," in 12th European Conference on Antennas and Propagation (EuCAP 2018), 2018, pp. 1-5.

[25] D. J. Sheskin, Handbook of parametric and nonparametric statistical procedures. CRC Press, 2003.

[26] D. A. Dickey and W. A. Fuller, "Distribution of the estimators for autoregressive time series with a unit root," Journal of the American Statistical Association, vol. 74, no. 366a, pp. 427-431, 1979.

[27] G. E. Box, G. M. Jenkins, G. C. Reinsel, and G. M. Ljung, Time series analysis: forecasting and control. John Wiley \& Sons, 2015.

[28] R. Nau, "Statistical forecasting: notes on regression and time series analysis," Durham: Fuqua School of Business, Duke University, 2015.

[29] J. E. Cavanaugh et al., "Unifying the derivations for the Akaike and corrected Akaike information criteria," Statistics \& Probability Letters, vol. 33, no. 2, pp. 201-208, 1997.

[30] S. L. Cotton, G. A. Conway, and W. G. Scanlon, "A time-domain approach to the analysis and modeling of on-body propagation characteristics using synchronized measurements at $2.45 \mathrm{GHz}$," IEEE Transactions on Antennas and Propagation, vol. 57, pp. 943-955, 2009.

[31] S. Das, Time series analysis. Princeton University Press, Princeton, NJ, 1994.

[32] G. M. Ljung and G. E. Box, "On a measure of lack of fit in time series models," Biometrika, vol. 65, no. 2, pp. 297-303, 1978

[33] J. Davidson, Econometric theory. Wiley-Blackwell, 2000.

[34] R. J. Hyndman and A. B. Koehler, "Another look at measures of forecast accuracy," International Journal of Forecasting, vol. 22, no. 4, pp. 679688,2006

[35] D. G. Brennan, "Linear diversity combining techniques," Proceedings of the IRE, vol. 47, no. 6, pp. 1075-1102, 1959.

[36] S. K. Yoo, S. L. Cotton, W. G. Scanlon, and G. A. Conway, "An experimental evaluation of switched combining based macro-diversity for wearable communications operating in an outdoor environment,' IEEE Transactions on Wireless Communications, vol. 16, no. 8, pp. 5338-5352, 2017

[37] J. Choi, "On the macro diversity with multiple BSs to mitigate blockage in millimeter-wave communications," IEEE Communications Letters, vol. 18, no. 9, pp. 1653-1656, 2014.

[38] M. Abusubaih, J. Gross, S. Wiethoelter, and A. Wolisz, "On access point selection in IEEE 802.11 wireless local area networks," in Proceedings. 2006 31st IEEE Conference on Local Computer Networks, 2006, pp $879-886$.

[39] S. Sanayei and A. Nosratinia, "Antenna selection in MIMO systems," IEEE Communications Magazine, vol. 42, no. 10, pp. 68-73, 2004
[40] H.-C. Yang and M.-S. Alouini, "Performance analysis of multibranch switched diversity systems," IEEE Transactions on Communications, vol. 51, no. 5, pp. 782-794, 2003 .

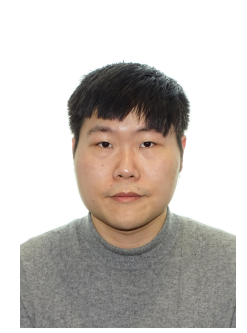

Lei Zhang received the B.Sc. degree in communication engineering from Shandong University, China, in 2012, and the M.Sc. degree in communication and information technology from University of Bremen, Germany, in 2016. He is currently pursuing the $\mathrm{Ph} . \mathrm{D}$. degree with the Centre for Wireless Innovation (CWI), ECIT Institute, Queen's University Belfast, Belfast, UK. His Doctoral studies were sponsored by the US-Ireland project. His research interests include mmWave channel modeling and measurements, machine learning for wireless communications, fading characterization for body-centric communications, and $5 \mathrm{G}$ wireless communications.

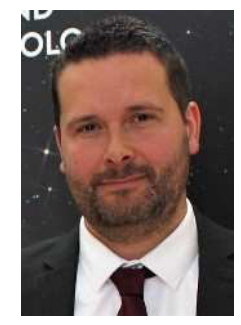

Simon L. Cotton (S'04-M'07-SM'14) received the B.Eng. degree in electronics and software from U1ster University, Ulster, U.K., in 2004, and the Ph.D. degree in electrical and electronic engineering from the Queen's University of Belfast, Belfast, U.K. in 2007. From 2007 to 2011 he was a Research Fellow, then Senior Research Fellow, 2011 to 2012, Lecturer (Assistant Professor), 2012 to 2015, and Reader (Associate Professor), 2015 to 2019 at the Queen's University of Belfast. He is currently a Full Professor and the Director of the Centre for Wireless Innovation (CWI) at Queen's University Belfast. Professor Cotton has authored and co-authored over 150 publications in major IEEE/IET journals and refereed international conferences, two book chapters, and two patents. Among his research interests are cellular device-to-device, vehicular, and body-centric communications. His other research interests include radio channel characterization and modeling, and the simulation of wireless channels. Professor Cotton was awarded the H. A. Wheeler Prize, in July 2010, by the IEEE Antennas and Propagation Society for the best applications journal paper in the IEEE TRANSACTIONS ON ANTENNAS AND PROPAGATION during 2009. In July 2011, he was awarded the Sir George Macfarlane Award from the U.K. Royal Academy of Engineering in recognition of his technical and scientific attainment since graduating from his first degree in engineering.

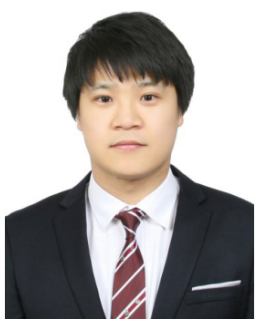

Seong Ki Yoo (S'14-M'17) received the B.Eng. (Hons.) degree in telecommunication systems from the University of Surrey, Guildford, U.K., in 2010 and the M.Sc. degree in communications and signal processing from Imperial College London, London, U.K., in 2012 and the Ph.D. degree from Queen's University Belfast, Belfast, U.K., in 2017. His Doctoral studies were sponsored by U.K. EPSRC. He was a junior researcher with Korea Electrotechnology Research Institute (KERI), Ansan, South Korea, from 2012 to 2013. He is currently a Lecturer in the School of Computing, Electronics and Mathematics, Coventry University. His research interests include mmWave channel modelling and measurements, fading characterization for wearable and vehicular communications channels and performance analysis over fading channels.

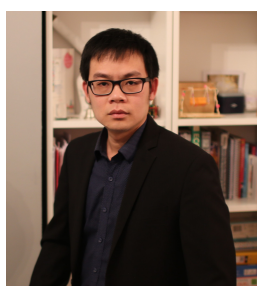

Hien Quoc Ngo is currently a Lecturer with Queen's University Belfast, U.K. He has coauthored many research articles in wireless communications and coauthored the Cambridge University Press textbook Fundamentals of Massive MIMO (2016). His main research interests include massive (large-scale) MIMO systems, cell-free massive MIMO, physical layer security, and cooperative communications. $\mathrm{He}$ received the IEEE ComSoc Stephen O. Rice Prize in Communications Theory in 2015, the IEEE ComSoc Leonard G. Abraham Prize in 2017, and the Best Ph.D. Award from EURASIP in 2018. He also received the IEEE Sweden VT-COM-IT Joint Chapter Best Student Journal Paper Award in 2015. He was awarded the UKRI Future Leaders Fellowship in 2019. He serves as the Editor for the IEEE Transactions on Wireless Communications, IEEE Wireless Communications Letters, the Digital Signal Processing, and the Physical Communication (Elsevier). He was a Guest Editor of IET Communications, and a Guest Editor of IEEE ACCESS in 2017. 


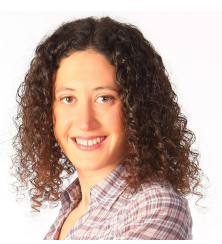

Marta Fernández (S'16-M'18) received the M.Sc. and Ph.D. in Telecommunications Engineering from the University of the Basque Country (Spain) in 2013 and 2018 respectively. She belongs to TSR (Radiocommunications and Signal Processing) research group at University of the Basque Country since 2013, where she is currently a Lecturer. In 2016 she was awarded a PANTHER (Erasmus Mundus) scholarship for visiting the Centre for Wireless Monitoring and Applications of Griffith University, Australia, where she spent 8 months undertaking part of her doctoral studies. In 2019, she was awarded a scholarship from the Spanish Government to conduct a 3-month research stay at Queens University Belfast, United Kingdom. She is currently the vice-chair of the IEEE BTS Spanish Chapter and the IEEE BTS Young Professional Cochair. Her research interests include wireless systems, radio frequency channel characterization and antenna design.

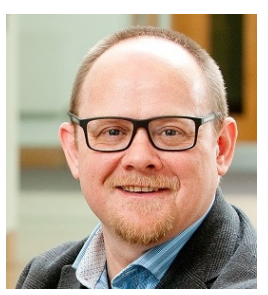

William G. Scanlon (M'98-SM'13-F'20) is Chief Executive Officer of Tyndall National Institute, Ireland. He was Senior Lecturer and Chair/Full Professor at Queen's University of Belfast (UK, 20022018) and held various leadership positions such as Director of the Centre for Wireless Innovation and Head of School of Electronics, Electrical Engineering and Computer Science. He held a parttime Chair in Short Range Radio at the University of Twente, The Netherlands (2009-2014). Scanlon is a pioneer in wearable and implantable medical device communications. He has served as keynote speaker for the International Symposium on Antennas and Propagation (2018), the IEEE Intl. Microwave Workshop Series on RF and Wireless Technologies for Biomedical and Healthcare Applications (2014), the NATO Military Communications and Information Systems Conf. (2010), the Intl. Conf. on Bodynets (2010 and 2018) and the European Workshop on Conformal Antennas (2007). He was a Series Editor of the IET Book Series on Telecommunications and Networking, an inaugural Associate Editor of IEEE Journal of Translational Engineering in Health and Medicine and an Associate Editor for IEEE Antennas and Wireless Propagation Letters. He holds an URSI Young Scientist award (1999), the 2010 IEEE H. A. Wheeler Prize Paper Award for IEEE Trans. Antennas and Propagation and he delivered the 2012 NATO Intl. Lecture Series on Next Generation Communications. 